\title{
An Effective Identification Systems Based on Iris Texture Analysis
}

\author{
Hossein Mohammadi, Mohammad Mohammadi, and Mahdieh Nadisenjani
}

\begin{abstract}
Iris identification has received extremely attention as a reliable approach to human identification in the world today. It is very effective for person identification due to the iris' unique features and the protection of the iris from the environment and aging. In this paper, we present a simple and effective method to create an iris recognition system by considering biological characteristics of iris where we used artificial neural networks for iris pattern recognition. The proposed approach is verified and it showed good results in terms of false acceptance ratio, false rejection ratio and noise immunity. In addition, the developed algorithm can uses for the another biometrics like fingerprint and retina.
\end{abstract}

Index Terms - Iris recognition, feature extraction, iris code, neural networks, multi layer perceptron, back propagation error.

\section{INTRODUCTION}

In the today's computerized world the biometrics are playing a fundamental role for the identification of persons. Biometrics makes use of certain physiological and behavioural characteristics of a person for identification or verification. These characteristics are called biometric modalities or traits. Fingerprint, iris, retina, face, hand geometry are samples of behavioural biometric modalities and signature, voice, gait, and keystroke dynamics are samples of physical biometric modalities[1].

Recently, instead of using traditional methods for personal identification such as PIN numbers, passwords and smart cards that can be lost, stolen or duplicated, biometrics is used in many applications to allow authorized person to enter a restricted place and to identify/verify a person. Biometric recognition systems use physical and/or behavioral characteristics that are unique and cannot be lost or forgotten [2].

Iris identification is one of the most popular and reliable and accurate personal characteristics for authentication process available today. The iris is so unique that no two irises are alike, even among identical twins, in the entire human population.

While most biometric have 13 to 60 distinct characteristics, the iris is said to have 400 unique spots.

Manuscript received September 13, 2012; revised November 26, 2012.

H. Mohammadi is with the Department of Electrical Engineering, Islamic Azad University, Sarvestan branch, Sravestan, Iran (e-mail: amir200@engineer.com).

M. Mohammadi is with the Department of Computer Engineering, Islamic Azad University, Dezful branch, Dezful, Iran (e-mail: mofard@gmail.com).

M. Nadisenjani is with the Computer Engineering Department, Islamic Azad University, Ashtian branch, Ashtian, Iran (e-mail: nadi.mahdieh@gmail.com).
Iris is a muscle within the eye that regulates the size of the pupil, controlling the amount of light that enters the eye and it is a protected internal organ whose random texture is stable throughout life, it can serve as a kind of living password that one need not remember but one always carries along. Since the degree of freedom of iris textures is extremely high, the probability of finding two identical irises is close to zero, and it cannot be tampered easily, therefore the iris recognition systems are very reliable [3].

In this paper, a simple and reliable method is proposed with the aim to recognize and identify iris texture. For avoid from long operation we prevented from the comparison between two adverse samples. Based on one of the existent methods we classified the Irises and put similar samples in same group as all Irises in each group are similar together and constituted a database. To find a query sample in this database, at first we find the group that the sample belongs to it. Then we start our search in the related group. For each process we employed a neural network. The major steps of proposed Iris identification are shown in Fig. 1.

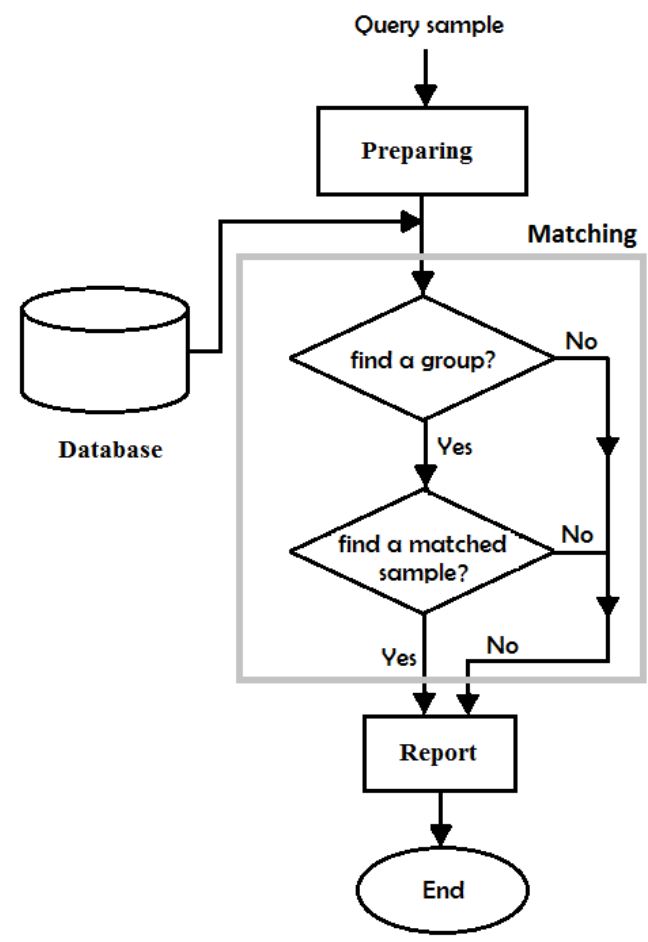

Fig. 1. The major steps of proposed iris identification.

\section{FEATURE EXTRACTION}

Our proposed method consists of two major steps. First step is the feature extraction and the second step is the verification $\&$ identification. In the first step we extracted the 
features in a numeric format called iris code. In the second step we designed a neural network which capable of accepting above iris codes as an input. The neural network compares iris code of an individual with the iris codes from the enrolled iris code database to determine a match or mismatch.

As we know, an identification system should extract and compare a feature unique to each iris. Since the texture pattern in each iris is unique, its pixel matrix is correspondingly unique. Therefore we can distinguish an iris from another with respect to its pixel matrix.

In the iris code the value of each number is proportional to the brightness of corresponding point in the scene. Its value is usually derived from the output of an analog to digital $(A / D)$ converter or digital scanner. An Iris image can describe as $M \times N$ m-bit pixels, where $M$ and $N$ are the number of points in each dimension and defined by sampling rate and $m$ controls the number of brightness values [4]. Using $\mathrm{m}$ bits gives a range of $2^{m}$ values, ranging from 0 to $2^{m-1}$. If $m$ is 8 , this gives brightness level ranging between 0 and 255, which are usually displayed as black and white, respectively, with shades of gray in between, as they are for the greyscales image but for binary images $\mathrm{m}$ is 2 . Therefore brightness levels can be only 0 or 1 . An iris with its iris code is shown in Fig. 2.

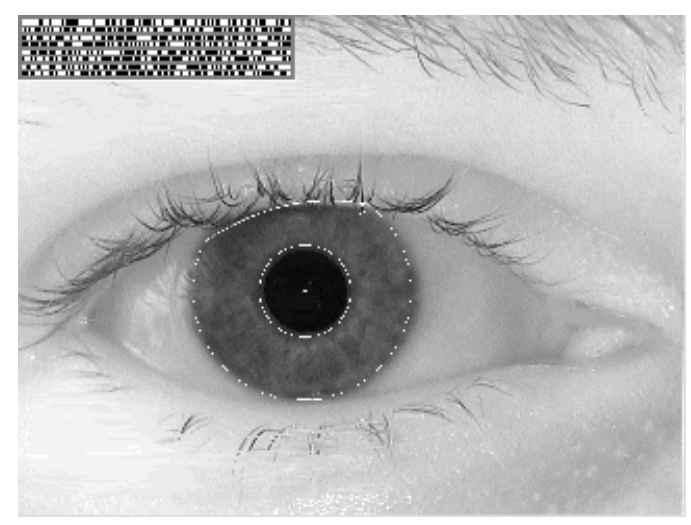

Fig. 2. An iris image with its iris code.

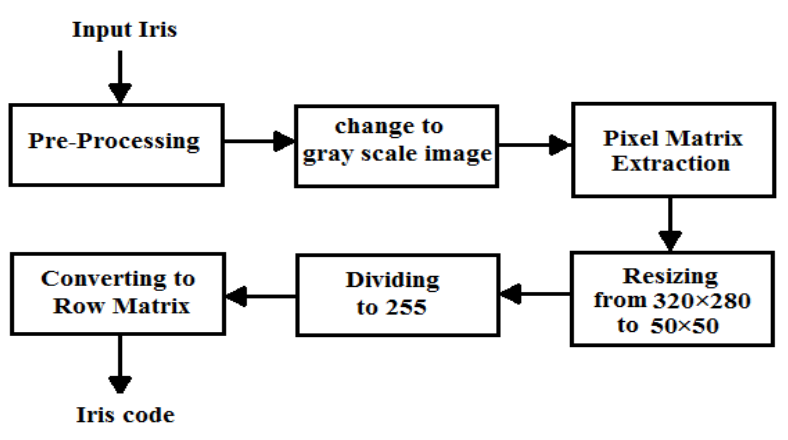

Fig. 3. The process of feature extraction.

We used CASIA database to analysis our method. The resolution of each image in this database is $320 \times 280$. Then we converted the input color images to grey scale and changed its dimension to $50 \times 50$ to reduce the time processing Then we divided it to 255 for making a matrix with its elements between 0 and 1 . Then we put all rows alongside together and made a row matrix with $1 \times 2500$ dimension. This process is shown in Fig. 3. We repeated the aforementioned steps for all the desired samples and saved them in a matrix with $n \times(2500)$ dimension where $n=80$ is the number of Irises which used as a training pattern in neural network.

For using another biometrics like fingerprint we should extract fingerprint code from the fingerprint images and use them as a network training algorithm.

\section{ThE PRoposed AlgorithM}

\section{A. Use of Neural Networks}

Artificial neural networks (ANNs) provide a general practical method for real-valued, discrete-valued, and vector-valued functions from examples. The Algorithm back propagation which is widely used in ANNs uses gradient descent to tune network parameters to best fit a training set of input-output pairs. Neural networks use training algorithms, such as back propagation, to learn the relationship between the presented data. Non-numeric data needs to be represented in numeric form to use in a network, as the training data set is comprised of input and output values. By training the data, adjustments are made in the network and iteratively decrease the error obtained in the predictions. In this manner, the network will learn to simulate the function, at which point it can then be used to interpret new data to classification and identification [5].

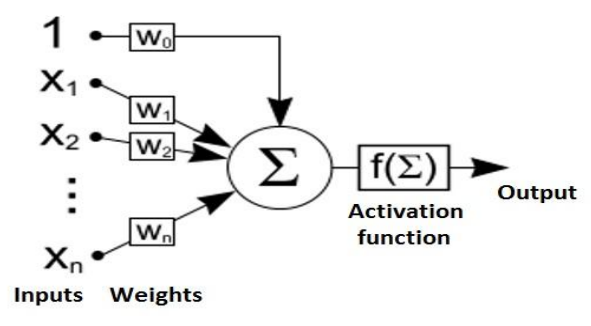

Fig. 4. Artificial neuron model.

Pattern recognition is formally defined as the process whereby a received pattern/signal is assigned to one of a prescribed number of classes (categories). A neural network performs pattern recognition by first undergoing a training session, during which the network is repeatedly presented a set of input patterns along with the category to which each particular pattern belongs. Later, a new pattern is presented to the network that has not been seen before, but which belongs to the same population of patterns used to train the network. The network is able to identify the class of that particular pattern because of the information it has extracted from the training data. Pattern recognition performed by a neural network is statistical in nature, with the patterns being represented by points in a multidimensional decision space. The decision space is divided into regions, each one of which is associated with a class. The decision boundaries are determined by the training process. The construction of these boundaries is made statistical by the inherent variability that exists within and between classes.

In our system, we used a multilayer perceptron for the problem. The first layer is comprised of 2500 units dealing with the input vector. The hidden layer is comprised of 60 units, and the output layer has 30 units dealing with the class 
number. The network has trained to give an output between 1 and 30. This means that each output indicates the number of group. For example, if the desired sample is from the first group, the first output equals 1 and the other outputs equal -1 so the output vector is $[1,-1,-1, \ldots,-1]$. Then we entered all iris codes to the neural network and trained it for group identification. For matching in each group we used another multi layer perceptron that its first layer is comprised of 2500 units dealing with the input vector; its hidden layer is comprised of 8 units, and its output layer is comprised of 4 units deal with the iris number. The network was trained to give an output between 1 and 4. Each output indicates the number of iris code in its group. For example if the desired sample is first one in its group, the first output equals 1 and the other outputs equal -1 so the output vector is $[1,-1,-1,-1]$. We used the back propagation error algorithm to train the network.

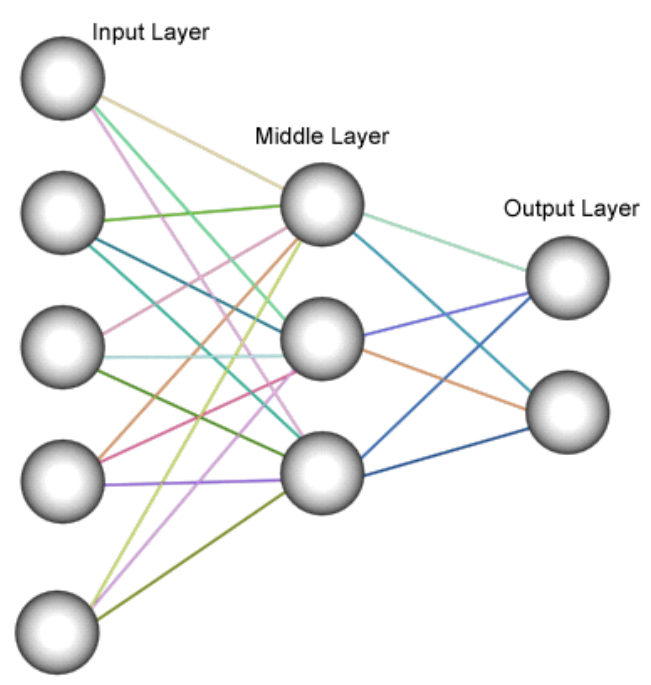

Fig. 5. Multilayer perceptron

\section{B. Training of Neural Networks}

Among the many interesting properties of a neural network is the ability of the network to learn from its environment and to improve its performance through learning. A neural network learns about its environment through an iterative process of adjustments applied to its synaptic weights and thresholds. The training process requires a set of examples of correct network behaviour that contains network inputs and desired outputs. During the training, the weights and biases of the network are adjusted iteratively to optimize the network performance function. The algorithm for training of neural networks consists of the following steps:

1) Initialize the network: Set all weights to random numbers between -1 and +1 and set thresholds to small random values.

2) Present the first training pattern (input and desired output)

3) Calculate the actual output: Each layer should be calculated as following:

$$
y_{o}=F\left[w_{0} x_{0}+w_{1} x_{1}+\ldots+w_{n} x_{n}\right]
$$

Then, this passes to the next layer as an input. Then we compare the network output with the target output.

4) Adapts weights: Starting from the output we propagate the error backwards. Correct the output layer of weights using the following formula.

$$
w_{h o}=w_{h o}+\left(\eta \delta_{o} o_{h}\right)
$$

where $w_{h o}$ is the weight connecting hidden unit h with output unit $\mathrm{o}, \eta$ is the learning rate, $\mathrm{o}_{\mathrm{h}}$ is the output at hidden unit $\mathrm{h}$. $\delta_{\mathrm{o}}$ is given by the following.

$$
\delta_{o}=o_{o}\left(1-o_{o}\right)\left(t_{o}-o_{o}\right)
$$

where $o_{o}$ is the output at node o of the output layer and $\mathrm{t}_{\mathrm{o}}$ is the target output for that node.

Correct the input weights using the following formula.

$$
w_{i h}=w_{i h}+\left(\eta \delta_{h} o_{i}\right)
$$

where $w_{i h}$ is the weight connecting node $\mathrm{i}$ of the input layer with node $h$ of the hidden layer, $\mathrm{o}_{\mathrm{i}}$ is the input at node $\mathrm{i}$ of the input layer, $\eta$ is the learning rate. $\delta_{\mathrm{h}}$ is calculated as following.

$$
\delta_{h}=o_{h}\left(1-o_{h}\right) \sum_{o} \delta_{o} w_{h o}
$$

\section{EXPERIMENTAL RESULTS}

To demonstrate the efficiency of our method, the proposed system has been developed using the MATLAB Prototyping environment but it can be used in any programming language. Our experiment was performed on CASIA database that includes 108 classes and each class has 7 iris images captured in two sessions with a time interval about a month [6]. So there are totally 756 iris images with a resolution of $320 \times 280$ pixels [4]. Two sample images in CASIA database are shown in Fig. 4. In our experiment we used 210 samples of this database in 30 groups.
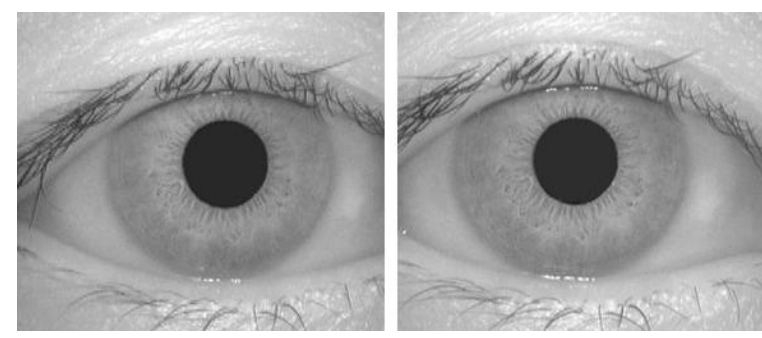

Fig. 6. Samples of iris images from CASIA database.

For a complete evaluation of the performance of our method we divided the experimental process in two parts. In the first part, performance of our algorithm has been measured in terms of false reject rate (FRR) and false accept rate (FAR). In the second part, we demonstrated the efficiency of our method in some parameters such as incomplete and noisy images.

\section{A. False Rejection Rate}

One of the most important specifications in any biometric system is the false rejection rate (FRR). The FRR is defined as the percentage of identification instances in which false rejection occurs. This can be expressed as a probability.

$\mathrm{FRR} \%=($ true claims rejected/total true claims $) \times 100[7]$ 
In this paper, the FRR is 0.96 percent, which means that 208 of the authorized persons attempting to access the system will be recognized by that system.

\section{B. False Acceptance Rate}

The false acceptance rate (FAR) is the measure of the likelihood that the biometric security system will incorrectly accept an access attempt by an unauthorized user. A system FAR typically is stated as the ratio of the number of false acceptances divided by the number of identification attempts. $\mathrm{FAR} \%=($ imposter claims accepted/total imposer claims $)$ $\times 100$ [7]

In this paper, the FAR is 0 percent, which means that no sample of 210 impostors (which used from another classes in CASISA database) attempting to breach the system will be successful. Stated another way, it means that the probability of an unauthorized person being identified an authorized person is 0 .

\section{Noise Immunity}

To verify the effect of noise on FAR and FRR we applied a salt and pepper noise (or spike noise) to some samples and varied its noise density from 0 to 0.1 (see Fig. 6.). An image containing salt and pepper noise will have dark pixels in bright regions and bright pixels in dark regions. This type of noise can be caused by dead pixels, analog-to-digital converter errors, bit errors in transmission, etc.

The result is shown in Fig. 8. It shows that this noise has no effect on FAR but FFR is changed from 0.96 (in Fig. 7-A) to 23(in Fig. 7-C).

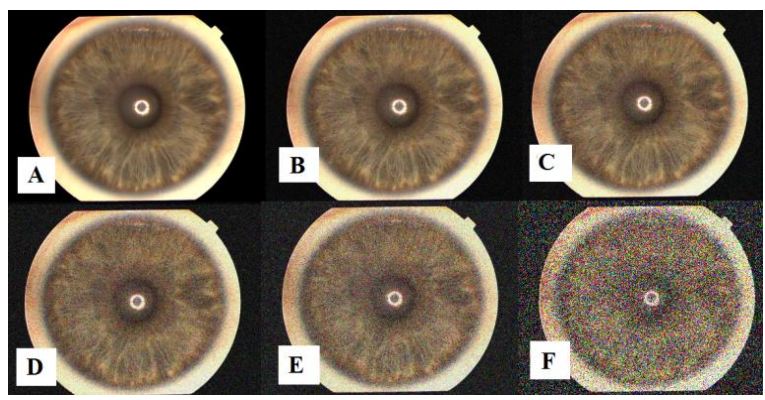

Fig. 7. Iris images mixed with salt and pepper in different noise intensity A: 0; B: $0.01 ;$ C: $0.02 ;$ D: $0.05 ; \mathrm{E}: 0.1 ; \mathrm{F}: 0.2$

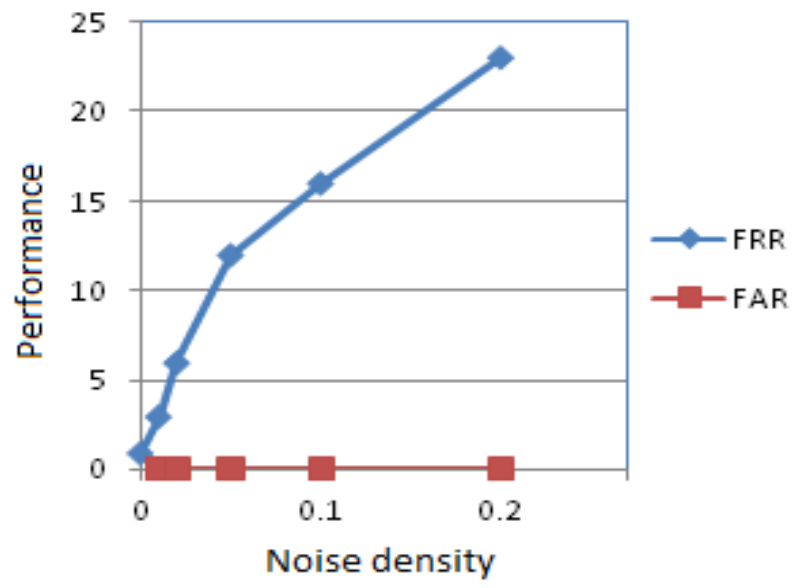

Fig. 8. The effect of noise on FRR and FAR

Table I describes the results of our test. References [8] and
[9] are compared with our method. From the results above, we know that our algorithm has an excellent recognition rate to incomplete and defiled iris images, and has strong robustness.

TABLE I: THE RESULTS OF OUR VERIFICATION

\begin{tabular}{llllll}
\hline \hline Method & $\begin{array}{l}\text { No. of } \\
\text { images }\end{array}$ & $\begin{array}{l}\text { True } \\
\text { Accept }\end{array}$ & $\begin{array}{l}\text { False } \\
\text { Reject }\end{array}$ & $\begin{array}{l}\text { True } \\
\text { Reject }\end{array}$ & $\begin{array}{l}\text { False } \\
\text { Accept }\end{array}$ \\
\hline Our approach & 210 & $99.04 \%$ & $0.96 \%$ & $100 \%$ & 0 \\
{$[8]$} & 150 & $98.9 \%$ & $1.08 \%$ & $100 \%$ & 0 \\
{$[9]$} & 432 & $98.84 \%$ & $1.16 \%$ & $99.77 \%$ & $0.23 \%$ \\
\hline \hline
\end{tabular}

\section{CONCLUSION}

Being able to correctly classify and identify irises for analysis is not a simple task. This particular study gives some wisdom into the use of neural networks for iris analysis. However, it is also evident that the sample size of the data involved needs to be increased as further study progresses.

Neural networks, much as many other efforts will never be $100 \%$ accurate. However they appear to be a suitable method to use in helping to classify and identify irises.

In this paper, we have successfully used back-propagation neutral network to implement an iris identification system with a proper fault tolerance. The experimental results have shown that the iris identification method is robust, reliable and rapid.

\section{REFERENCES}

[1] A. K. Jain, R. Bolle, and S. Pankanti, "Personal identification in networked society," Biometrics, Springer, vol. 2, 2006.

[2] A. Poursaberi and B. N. Araabi, "Iris recognition for partially occluded images: Methodology and sensitivity analysis," EURASIP Journal on Advances in Signal Processing, 2007.

[3] U. M. Chaskar and U. M. Chaskar, "A novel approach for iris recognition," 2nd International Conference on Computer Technology and Development (ICCTD 2010). pp. 495-500, 2010

[4] M. S. Nixon and A. S. Aguado, Feature Extraction and Image Processing, Elsevier, 2008

[5] Statessoft Inc. [Online]. Available: http://www.statsoftinc.com/textbook/stneunet.html $\neq$ suing, 1984-2003

[6] Center for Biometrics and Security Research (CBSR). CASIA Iris Image Database. [Online]. Available: http://www.sinobiometrics.com.

[7] J. Kour and N. Awasthy, "Nonminutiae based fingerprint matching," IEEE Computer Society, pp. 199-203, 2009

[8] S. R. Kodituwakku and M. I. M. Fazeen, "An offline fuzzy based approach for iris recognition with enhanced feature detection," Advanced Techniques in Computing Sciences and Software Engineering 2010, pp. 39-44

[9] M. Ramli, N. A. Kamarudin, M. Saufi, and J. Ariffuddin, "Iris recognition for personal identification," The International Conference on Electrical Engineering, Okinawa, Japan, 2008.

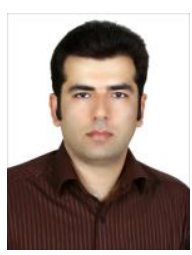

Hossein Mohammadi was born in Shiraz, Iran in 1974 He received his B.Sc in Electronic engineering from IAU of Iran, Dezful branch in 1999. He continued his education and finished his M.Sc in electronic faculty of IAU Arak branch in 2008. He is currently PhD student of National University of Malaysia (UKM) in Electrical, Electronics and systems department. He is currently academic member of faculty in IAU Sarvestan branch. His research interests include Neural Networks, Microelectronics and modeling. 


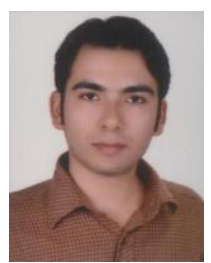

Mohammad Mohammadi was born in Tehran, Iran in 1986. He received his B. Sc in Computer engineering from IAU of Iran, Dezful branch in 2009. $\mathrm{He}$ is currently master student of IAU of Iran, Dezful branch in computer architecture. His research interests include Neural Networks and Network on Chip.

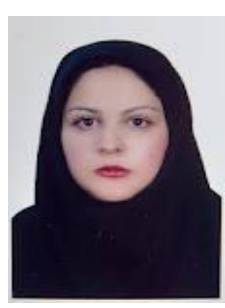

Mahdieh Nadisenjani was born in Arak, Iran. She received her B.Sc and M.Sc in computer engineering and computer architecture from IAU of Iran, Arak branch in 2005 and 2007 respectivly. She is currently $\mathrm{PhD}$ student of University Technology Malaysia (UTM) in computer science faculty (FSKSM). She is currently member of faculty in IAU Ashtian branch. Her research interests include power modeling, network on chip design and modeling. 